\title{
Rigid Symmetries and Conservation Laws in Non-Lagrangian Field Theory
}

\author{
D.S. Kaparulin, S.L. Lyakhovich and A.A. Sharapov \\ Department of Quantum Field Theory, Tomsk State University, \\ Lenin ave. 36, Tomsk 634050, Russia
}

\begin{abstract}
Making use of the Lagrange anchor construction introduced earlier to quantize non-Lagrangian field theories, we extend the Noether theorem beyond the class of variational dynamics.
\end{abstract}

\footnotetext{
* This work was partially supported by the RFBR grant 09-02-00723-a, by the grant from Russian Federation President Programme of Support for Leading Scientific Schools no 3400.2010.2., by the State Contract no 02.740.11.0238 from Russian Federal Agency for Science and Innovation, and also by Russian Federal Agency of Education under the State Contracts no P1337 and no P22. SLL appreciates partial support from the RFBR grant 08-01-00737-a.
} 


\section{Contents}

1 Introduction

2 Classical fields

3 Symmetries, identities and conservation laws 9

4 The Lagrange anchor 15

5 Proper symmetries and proper deformations 18

6 Field-theoretical examples 25

$6.1 p$-form fields . . . . . . . . . . . . . . . . . 25

6.2 Self-dual $p$-form fields . . . . . . . . . . . . . . . . . . 28

6.3 Chiral bosons in two dimensions . . . . . . . . . . . . . . . . . 29

7 Conclusion 31 


\section{Introduction}

In this paper we extend the Noether theorem beyond the class of variational dynamics.

The classical field theory is completely defined by equations of motion. While the least action principle is not vital at classical level, it provides a number of useful tools for studying classical dynamics. In particular, given an action functional enjoying infinitesimal symmetries, one can derive conserved currents. For non-variational equations, the infinitesimal symmetries do not necessarily result in the conservation laws and vice versa. All the symmetries are divided into gauge and rigid. The gauge symmetries are unambiguously connected to Noether's identities (also called the strong conservation laws) as far as equations are variational. If the equations do not come from the least action principle, this relationship (sometimes called the second Noether theorem) is generally invalid: It is possible to have gauge invariant field equations without Noether's identities or equations possessing strong conservation laws without being gauge invariant.

In the gauge field theory, the existence of action is a key prerequisite for constructing the standard BRST theory either in the Hamiltonian BFV form [1] or in the BV field-antifield formalism [2] (see [3] for review). The BRST theory, in its turn, provides the most general tools for quantizing gauge theories [1, 2, 3]. Also, the BRST formalism serves for the study of various classical problems such as constructing consistent interactions in gauge models [4] or identifying the nontrivial conservation laws and rigid symmetries [5].

Now, it is known [6] that the BRST theory can be extended beyond the class of dynamical systems whose equations of motion are variational. Classically, the BRST complex can always be constructed for any regular system of field equations, variational or not [3], [6], [7]. The quantization, however, requires an extra ingredient, besides the field equations. In the framework of the deformation quantization it is the weak Poisson structure [6] (see also [8]). The existence of the weak Poisson structure is much less restrictive for the evolutionary equations than the requirement to be Hamiltonian. In the framework of the path-integral quantization, the corresponding extra ingredient was first introduced in [7] and called the Lagrange structure. Again, the existence of the Lagrange structure is much less restrictive for the equations than the requirement to be variational or admit an equivalent variational reformulation. Whenever the Lagrange structure is known for a given system of equations, the theory can be consistently quantized in three equivalent ways. First, the original non-Lagrangian field theory in $d$ dimensions can be converted into equivalent Lagrangian topological field theory in $d+1$. The latter can be then quantized by the usual BV procedure [7]. Second, the generating functional of Green's functions can be 
defined through the generalized Schwinger-Dyson equation [9], [10]. Third, the original non-Lagrangian field theory can be embedded into an augmented Lagrangian theory in the same $d$ dimensions [11].

Besides providing the basis for quantizing non-Lagrangian field theories, the Lagrange structure binds together gauge symmetries and the strong conservation laws, although the relationship is more relaxed than for the variational case [7]. In the first-order formalism, for example, the constraints and gauge symmetries remain fully unrelated [12] unless the equations are Hamiltonian. A certain correlation is set up by the weak Poisson structure, although it is not so rigid as the relation between the first-class constraints and gauge symmetries in the Hamiltonian constrained dynamics.

In this paper we will show that the Lagrange structure allows one to connect the rigid symmetries with the conservation laws. This relationship reveals itself in a different way than the link between the gauge symmetries and the strong conservation laws in nonLagrangian theories equipped with the Lagrange structure. In the next sections we give accurate definitions for symmetries, identities and conservation laws and, by making use of the Lagrange structure, establish a relationship between them. The general construction is then exemplified by a class of non-Lagrangian models, where some fundamental symmetries and/or conserved currents can be explicitly found and compared.

To give a preliminary impression of the connection between the symmetries and conservation laws, below in the Introduction, we reformulate the standard Noether theorem in the way that illuminates the points which are important for extending the theorem beyond the class of Lagrangian dynamics.

Let $M$ denote the configuration space of fields $\varphi^{i}$. Hereinafter we use De Witt's condensed notation [17] whereby the index $i$, labelling the fields, includes also the space-time coordinates $\left\{x^{\mu}\right\}$. The smooth functions $f(\varphi)$ on $M$ are identified with the local functionals of fields. In terms of the condensed notation, the Lagrangian equations of motion are given by the components of an exact 1 -form $\mathrm{d} S$ on $M$, where $\mathrm{d}=\delta \varphi^{i} \frac{\delta}{\delta \varphi^{i}}$ is the variational differential and $S$ is the action functional. In general, the field equations can well be the components of a section of another bundle over $M$, not necessarily $T^{*} M$. For example, the tensor type of equations can differ from that of fields (see the next section). Here, however, we restrict our discussion to equations whose left hand sides are given by the components of a local 1-form $T=T_{i}(\varphi) \delta \varphi^{i}$ on $M$. Then the necessary condition for the existence of an action for the field equations $T_{i}(\varphi)=0$ requires $T$ to be a closed 1-form

$$
\mathrm{d} T=0 .
$$

This is the well-known Helmholtz criterion from the inverse problem of the calculus of 
variations.

Recall that a local vector field $\Psi=\Psi^{i}(\varphi) \frac{\delta}{\delta \varphi^{i}}$ on $M$ is called a characteristic of the equations $T_{i}=0$ if

$$
\mathrm{d}\left(i_{\Psi} T\right) \equiv 0, \quad i_{\Psi} T \equiv \Psi^{i} T_{i}
$$

Since the value $i_{\Psi} T$ is annihilated by the variational derivative, it must be the integral of a total divergence $\partial_{\mu} j^{\mu}$. By definition, the latter vanishes on the equations of motion, and hence $j^{\mu}$ is a conserved current.

Every vector field $\Psi$ generates a transformation $\delta \varphi^{i}=\Psi^{i}(\varphi)$ of the space of fields $M$ and we say that $\Psi$ is a proper symmetry of the equations of motion if

$$
L_{\Psi} T=0
$$

where $L_{\Psi}$ is the Lie derivative along $\Psi$. For the variational equations of motion $T=\mathrm{d} S$ the proper symmetries are clearly the symmetries in the usual sense. Making use of Cartan's formula

$$
L_{\Psi} T=i_{\Psi}(\mathrm{d} T)+\mathrm{d}\left(i_{\Psi} T\right),
$$

we see that the proper symmetry $\Psi$ is a characteristic if $i_{\Psi}(\mathrm{d} T)=0$. For the variational equations of motion, satisfying the Helmholtz condition (1.1), the last formula identifies symmetries with characteristics. It is the statement which is known as the Noether theorem.

Let us summarize the lessons that can be learned from the above formulation of the Noether theorem towards its extension beyond the scope of variational dynamics. The construction of characteristics from symmetries involves, besides the equations of motion, one more crucial ingredient, namely, the variational exterior differential $d$ that maps the variational $p$-forms to the $(p+1)$-forms. In the particular case discussed above, the equations of motion are given by 1 -forms. Three main properties of the differential $\mathrm{d}$ are relevant for establishing the link between the symmetries and conservation laws. First, the field equations are d-closed (1.1). Second, the exterior differential and the Lie derivative are related to each other through Cartan's formula (1.4). Third, the kernel of the variational differential is constituted by the integrals of total divergencies $\partial_{\mu} j^{\mu}$. These three facts taken together imply that the symmetries can be identified with the characteristics.

The general field equations need not be a section of the cotangent bundle $T^{*} M$. They may well be associated with another vector bundle $\mathcal{E} \rightarrow M$ over the configuration space of fields $M$, in which case the differential $\mathrm{d}$ does not work any more. Thus, one needs a more general operator $d_{\mathcal{E}}$ to replace the variational exterior differential in the case of non-Lagrangian equations of motion. The aforementioned Lagrange structure provides 
such an operator that satisfies the first two properties. The third one is obeyed, in a sense, only by weakly transitive Lagrange structures. Thus, whenever the non-Lagrangian field equations enjoy a weakly transitive Lagrange structure, the Noether theorem can be still formulated in full. In the intransitive case, a weaker proposition can be proved, connecting rigid symmetries with characteristics in a more relaxed way.

\section{Classical fields}

As is well known, the geometry underlying the cinematics of local field theory is that of jet bundles. So we begin the section with recalling some basics from the jet theory relevant for our purposes. For a systematic exposition of the subject the reader may consult the books [13, 14, 15, 16].

The starting point of any local field theory is a locally trivial fiber bundle $\pi: Y \rightarrow X$, which base $X$ is usually identified with the space-time manifold and which sections, i.e., the smooth maps $\varphi: X \rightarrow Y$ satisfying $p \circ \varphi=\operatorname{id}_{X}$, are called fields or histories. The space of all histories (= sections of $Y$ ) will be denoted by $M$. The typical fiber of $Y$, being given by $U \simeq \pi^{-1}(x), x \in X$, is usually referred to as the target space of fields.

The $r$-th jet prolongation of the fiber bundle $Y \rightarrow X$ is the bundle $\pi_{r}: J^{r} Y \rightarrow X$, whose points are $r$-jets. By definition, an $r$-jet $j_{x}^{r} \varphi$ is the equivalence class of a local section $\varphi$ of $Y$, where two local sections $\varphi$ and $\varphi^{\prime}$ are considered to be equivalent if they have the same Taylor development of order $r$ at $x \in X$ in some (hence any) pair of coordinate charts centered at $x$ and $\varphi(x)$. It follows from the definition that each section $\varphi$ of $Y$ induces the section $j^{r} \varphi$ of $J^{r} Y$ by the rule $\left(j^{r} \varphi\right)(x)=j_{x}^{r} \varphi$. If $Y$ is coordinatized by the numbers $\left\{x^{\mu}, u^{i}\right\}$, where $\left\{x^{\mu}\right\}$ and $\left\{u^{i}\right\}$ are local coordinates in $X$ and $U$, then $\left(x^{\mu}, u^{i}, u_{\mu_{1}}^{i}, u_{\mu_{1} \mu_{2}}^{i}, \ldots, u_{\mu_{1} \cdots \mu_{r}}^{i}\right)$ are local coordinates in $J^{r} Y$ and the section $j^{r} \varphi$ is given in these coordinates by

$$
x \mapsto\left(x, \varphi^{i}(x), \partial_{\mu_{1}} \varphi^{i}(x), \partial_{\mu_{1}} \partial_{\mu_{2}} \varphi^{i}(x), \ldots, \partial_{\mu_{1}} \ldots \partial_{\mu_{r}} \varphi^{i}(x)\right)
$$

Classical dynamics on $Y \rightarrow X$ are specified by imposing differential equations. An $r$-th order differential equation is, by definition, a closed imbedded submanifold $S \subset J^{r} Y$. A solution, or true history $\varphi$, is a section of $Y$ satisfying $j^{r} \varphi \in S$. The true histories form a subspace $\Sigma$ in the space of all histories $M$. In classical field theory, the differential equations are mostly defined by (nonlinear) differential operators associated to vector bundles. Let $E \rightarrow X$ be a vector bundle. The $r$-th order $E$-valued differential operator is a map 
$T: M \rightarrow \Gamma(E)$ such that the value of the section $T(\varphi)$ at $x \in X$ is fully determined by $j_{x}^{r} \varphi$. This definition assumes the existence of a bundle morphism $\Theta: J^{r} Y \rightarrow E$ such that $T(\varphi)=\Gamma(\Theta) \circ j^{r}(\varphi)$. The differential equation $S \in J^{r} Y$ is then identified with the pre-image of the base $X \subset E$, considered as the zero section, under the bundle map $\Theta$. In each local coordinate chart on $E \oplus J^{r} Y$, the condition that $\varphi$ belongs to the kernel of the map $T$ takes the form of partial differential equations

$$
T_{a}\left(x, \varphi^{i}, \partial_{\mu_{1}} \varphi^{i}, \partial_{\mu_{1}} \partial_{\mu_{2}} \varphi^{i}, \ldots, \partial_{\mu_{1}} \ldots \partial_{\mu_{r}} \varphi^{i}\right)=0,
$$

where $\left\{T_{a}\right\}$ are components of the section $T(\varphi)$ with respect to some frame $\left\{e^{a}\right\}$ in $E$.

Though rigor and geometrically transparent, the language of jets appears to be unduly cumbersome in developing general field-theoretical constructions where the local structure of fields, while important, is out of focus. For this reason we will use a little bit loose but much more handy notation known in the physical literature as De Witt's condensed notation [17]. According to this notation, the fields $\varphi^{i}(x)$ are treated as local coordinates on the infinite dimensional manifold $M$; in so doing, the index $i$ also includes the local coordinates $\left\{x^{\mu}\right\}$ on $X$ so that $\varphi^{i} \equiv \varphi^{i}(x)$. Similarly, the sections $s=s_{a}(x) e^{a}$ of the vector bundle $E \rightarrow X$ are considered to be linear coordinates $s_{a} \equiv s_{a}(x)$ on the infinite dimensional vector space $\Gamma(E)$. Then one can view the differential operator $T: M \rightarrow$ $\Gamma(E)$ as a section of the trivial, infinite dimensional vector bundle $\mathcal{E}=M \times \Gamma(E)$ over $M$. Of a particular importance for our consideration will be differential operators with values in differential forms on $X$, this is the case of $E=\wedge^{\bullet} T^{*} X$. We will denote the space of all such operators by $\Omega^{\bullet}$. The scalar differential operators are, by definition, the elements of the space $\Omega^{0}$. Let $\nu$ be a fixed volume form on $X$. By smooth functions on $M$ we understand the local functionals of fields. These are given by integrals

$$
f[\varphi]=\int_{X} \nu F(\varphi)
$$

of scalar differential operators $F$ evaluated for fields $\varphi \in M$ of compact support. The relation between local functionals and scalar differential operators is not generally one-toone. For example, if $\partial X=\varnothing$ and $F$ and $F^{\prime}$ are two scalar operators such that $\nu\left(F^{\prime}-F\right)=$ $d j$ for some $j \in \Omega^{n-1}, n=\operatorname{dim} X$, then $f^{\prime}=f$. We say that two local functionals $f$ and $f^{\prime}$ are equivalent modulo boundary terms, $f \doteq f^{\prime}$, if they differ by a local functional of the form $\int_{X} d j(\varphi)$. By the Stokes theorem the equivalence implies that $f^{\prime}-f=\int_{\partial X} j(\varphi)$.

In the sequel, for the sake of simplicity, we will assume that $Y \rightarrow X$ is a trivial vector bundle over a contractible domain in $\mathbb{R}^{n}$. Then, one has a simple criterion for the local functional $f$ to vanish modulo boundary terms [3], [5], [15]:

$$
\mathrm{d} f \equiv \int_{X} \nu \frac{\delta f}{\delta \varphi^{i}} \delta \varphi^{i}=0 \quad \forall \varphi \quad \text { iff } \quad f \doteq 0 .
$$


Of course, in a topologically nontrivial situation only the "if" part of the criterion holds true. Following the finite dimensional pattern, we can interpret the variations $\mathrm{d} f$ of local functionals as exact 1 -forms on $M$, with $\left\{\delta \varphi^{i}\right\}$ being a local frame in $T^{*} M$. The components of the 1 -form $\mathrm{d} f$ define the Euler-Lagrange differential operator $\delta f / \delta \varphi^{i}$. In a similar manner, one can introduce the tangent bundle $T M$ as the vector bundle which sections are evolutionary vector fields

$$
V=\int_{X} \nu V^{i}\left(x, \varphi, \ldots, \partial_{\mu_{1}} \ldots \partial_{\mu_{r}} \varphi\right) \frac{\delta}{\delta \varphi^{i}(x)}
$$

Every evolutionary vector field $V$ generates a flow $\Phi_{t}^{V}$ on the space of all histories $M$, which is described by the evolution-type system $\partial_{t} \varphi^{i}=V^{i}$ of PDEs, hence the name.

Given a fiber bundle $Y \rightarrow X$, the correspondence $E \mapsto \mathcal{E}$ is quite natural in the sense that it allows one to extend all the usual tensor operations from the finite dimensional vector bundles to the corresponding infinite dimensional ones. In particular, one can define the dual of the vector bundle $\mathcal{E}$ as the vector bundle $\mathcal{E}^{*}$ corresponding to $E^{*}$. There is a natural pairing between sections of $\mathcal{E}$ and $\mathcal{E}^{*}$ defined by the integral

$$
\langle S, T\rangle=\int_{X} \nu\langle S(\varphi), T(\varphi)\rangle \quad \forall S \in \Gamma\left(\mathcal{E}^{*}\right), \forall T \in \Gamma(\mathcal{E}) .
$$

As is seen the result of pairing is a smooth function on $M$. In accordance with De Witt's convention, we can write the last expression just as the sum $S^{a} T_{a}$, where the repeated index $a$ implies also integration over $X$.

Each homomorphism $h: E \rightarrow E^{\prime}$ induces the homomorphism $H: \mathcal{E} \rightarrow \mathcal{E}^{\prime}$ of the associated vector bundles through the action on their fibers, $\Gamma(h): \Gamma(E) \rightarrow \Gamma\left(E^{\prime}\right)$. But the infinite dimensional vector bundles $\mathcal{E} \rightarrow M$ admit more general homomorphisms. By definition, a general homomorphism $H \in \operatorname{Hom}\left(\mathcal{E}, \mathcal{E}^{\prime}\right)$ is given by a differential operator

$$
H: \Gamma(Y \oplus E) \rightarrow \Gamma\left(E^{\prime}\right)
$$

such that the section $H(\varphi, s) \in \Gamma\left(E^{\prime}\right)$, where $\varphi \in M$ and $s \in \Gamma(E)$, depends $\mathbb{R}$-linearly on the second argument. As usual the homomorphism $H$ induces the homomorphism on sections. Namely, $\Gamma(H)$ takes a section $(\varphi, T(\varphi)) \in \Gamma(Y \oplus E)$ of $\mathcal{E}$ to the section $H(\varphi, T(\varphi))$ of $\mathcal{E}^{\prime}$.

We define the transpose of a homomorphism $H \in \operatorname{Hom}\left(\mathcal{E}_{1}, \mathcal{E}_{2}\right)$ as a unique homomorphism $H^{*} \in \operatorname{Hom}\left(\mathcal{E}_{2}^{*}, \mathcal{E}_{1}^{*}\right)$ satisfying the property

$$
\langle\Gamma(H)(W), P\rangle \doteq\left\langle W, \Gamma\left(H^{*}\right)(P)\right\rangle
$$


for all $W \in \Gamma\left(\mathcal{E}_{1}\right)$ and $P \in \Gamma\left(\mathcal{E}_{2}^{*}\right)$.

The direct product $\mathcal{E}_{1} \oplus \mathcal{E}_{2}$ is defined to be the bundle corresponding to $E_{1} \oplus E_{2}$. Finally, we define the tensor product

$$
\mathcal{E}_{0} \otimes \mathcal{E}_{1} \otimes \cdots \otimes \mathcal{E}_{m}
$$

as the trivial vector bundle over $M$ whose sections are differential operators

$$
H: \Gamma\left(Y \oplus E_{1}^{*} \oplus \cdots \oplus E_{m}^{*}\right) \rightarrow \Gamma\left(E_{0}\right)
$$

that are linear in the sections of $\Gamma\left(E_{k}^{*}\right), k=1, \ldots, m$. In case $m=1$, we have the usual isomorphism $\Gamma\left(\mathcal{E}_{0} \otimes \mathcal{E}_{1}\right) \simeq \operatorname{Hom}\left(\mathcal{E}_{1}^{*}, \mathcal{E}_{0}\right)$. Applying this construction to the cotangent bundle $T^{*} M$, we define the $p$-th exterior power of the cotangent bundle $\wedge^{p} T^{*} M$, whose sections are $p$-forms on $M$. The operator of variational derivative mentioned above gives rise to the exterior differential $\mathrm{d}: \Gamma\left(\wedge^{p} T^{*} M\right) \rightarrow \Gamma\left(\wedge^{p+1} T^{*} M\right)$ with the property $\mathrm{d}^{2}=0$.

One can view the theory of jet bundles as a "differential geometry with higher derivatives". The advantage of the condensed notation over jets is that it brings the subject back into the more familiar geometric framework without higher derivatives at the cost of passing to infinite dimensional manifolds. Of course, care must be exercised when using the standard differential-geometric constructions in the infinite dimensional setting. It is particularly important to keep in mind that unlike the finite dimensional case, the space of sections $\Gamma(\mathcal{E})$ is not a module over the space of smooth functions $C^{\infty}(M)$ (the latter consists of local functionals). Although it is possible to think of $\Gamma(\mathcal{E})$ as a module over the scalar differential operators $\Omega^{0}$ with respect to the pointwise multiplication of functions on $X$, the induced action of $\operatorname{Hom}\left(\mathcal{E}, \mathcal{E}^{\prime}\right)$ on $\Gamma(\mathcal{E})$ is only $\mathbb{R}$-linear, not $\Omega^{0}$-linear. Finally, $\Gamma\left(\mathcal{E}_{1} \otimes \mathcal{E}_{2}\right) \neq \Gamma\left(\mathcal{E}_{1}\right) \otimes \Gamma\left(\mathcal{E}_{2}\right)$.

\section{Symmetries, identities and conservation laws}

The discussion of the previous section can be summarized by saying that the classical dynamics of fields are fully specified by a section $T$ of some vector bundle $\mathcal{E} \rightarrow M$ over the space of all histories. For this reason we call $\mathcal{E}$ the dynamics bundle. The subspace of true histories $\Sigma \subset M$ is defined to be the zero locus of $T$,

$$
\Sigma=\{\varphi \in M \mid T(\varphi)=0\} .
$$

Using the physical terminology, we will refer to $\Sigma$ as the shell. The field equations $T(\varphi)=$ 0 are supposed to satisfy the standard regularity conditions usually assumed for PDEs, see 
e.g. [3], [5], [14]. These conditions ensure that any section $S \in \Gamma\left(\mathcal{E}^{\prime}\right)$ vanishing on $\Sigma$ is representable in the form

$$
S=\Gamma(H)(T)
$$

for some homomorphism $H \in \operatorname{Hom}\left(\mathcal{E}, \mathcal{E}^{\prime}\right)$.

The present section contains no original results, we just explain our terminology and briefly run through the notions of symmetry, identities and conservation laws, which are studied in the next sections.

To begin with, we introduce the homomorphism $J: T M \rightarrow \mathcal{E}$ defined by 1

$$
\langle J(X), \Psi\rangle=X \cdot\langle T, \Psi\rangle \quad \forall X \in \Gamma(T M), \quad \forall \Psi \in \Gamma\left(E^{*}\right),
$$

$\Psi$ being understood as a field independent section of $\mathcal{E}^{*}$. The homomorphism $J$ is called [16] the universal linearization of the nonlinear differential operator $T$. We also introduce the sign of weak equality $S \approx S^{\prime}$ borrowed from Dirac's constrained dynamics. It means that two sections $S$ and $S^{\prime}$ of some vector bundle over $M$ coincide on shell, i.e., $\left.S\right|_{\Sigma}=\left.S^{\prime}\right|_{\Sigma}$.

An evolutionary vector field $X$ is said to be a symmetry of the classical system if it preserves the shell $\Sigma$. This amounts to the weak equality

$$
J(X) \approx 0
$$

The symmetries form a subalgebra $\operatorname{Sym}^{\prime}(T) \subset \Gamma(T M)$ in the Lie algebra of all vector fields on $M$.

A section $\Psi \in \Gamma\left(\mathcal{E}^{*}\right)$ is said to be an identity of the classical system if

$$
\langle\Psi, T\rangle \doteq 0
$$

As a differential consequence of the last equality we have

$$
J^{*}(\Psi) \approx 0
$$

Denote by $\operatorname{Id}^{\prime}(T) \subset \Gamma\left(\mathcal{E}^{*}\right)$ the subspace of all identities.

The notions of symmetry and identity can be further elaborated on. Observe that any vector field $X$ that vanishes on the shell, i.e., $X \approx 0$, is a symmetry in the sense of (3.4). The on-shell vanishing symmetries are called trivial. They are present in any classical theory, containing no valuable information about the structure of dynamics. Fortunately,

\footnotetext{
${ }^{1}$ By abuse of notation, we will use the same symbol to denote a vector bundle homomorphism and the induced homomorphism on sections. In particular, we will write $J$ instead of more pedantic $\Gamma(J)$.
} 
being proportional to the equations of motion, the trivial symmetries constitute an ideal $\operatorname{Sym}_{0}(T) \subset \operatorname{Sym}^{\prime}(T)$ in the Lie algebra of all symmetries and can thus be systematically disregarded. So in what follows, by a symmetry we will actually mean an element of the factor algebra $\operatorname{Sym}(T)=\operatorname{Sym}^{\prime}(T) / \operatorname{Sym}_{0}(T)$.

A symmetry $X \in \operatorname{Sym}(T)$ is called a gauge symmetry if there exists a vector bundle $\mathcal{F} \rightarrow M$ together with a section $\varepsilon \in \Gamma(\mathcal{F})$ and a homomorphism $R \in \operatorname{Hom}(\mathcal{F}, T M)$ such that $\operatorname{Im} R \subset \operatorname{Sym}(T)$ and $X=R(\varepsilon)$. If the vector bundle $\mathcal{F}$ is big enough to accommodate any (nontrivial) gauge symmetry with a fixed $R$, then we refer to $\mathcal{F}$ as the gauge algebra bundle. Notice that $J \circ R \approx 0$. The homomorphism $R$ defines an (over)complete basis of gauge generators. Clearly, the vector distribution $\operatorname{Im} R \subset T M$ is on-shell involutive, and hence it defines a foliation of $\Sigma$. The leaves of this foliation are called gauge orbits. Two points $p, q \in \Sigma$ are considered to be (gauge) equivalent, $q \sim p$, if they belong to the same gauge orbit. The quotient space $\Sigma / \sim$ is termed as a covariant phase space. It is the space of all physical states of the system.

Since the gauge symmetries form an ideal $\operatorname{GSym}(T) \subset \operatorname{Sym}(T)$, one can define the factor algebra $\operatorname{RSym}(T)=\operatorname{Sym}(T) / \operatorname{GSym}(T)$, which is naturally identified with the Lie algebra of all rigid symmetries. By definition, the rigid symmetries are transverse to the gauge orbits and thus they induce a nontrivial action on the phase space of physical states.

Equation (3.5) admits a lot of trivial solutions that can be constructed as follows. Take an arbitrary section of $K \in \Gamma\left(\mathcal{E}^{*} \wedge \mathcal{E}^{*}\right)$ and consider it as a homomorphism from $\operatorname{Hom}\left(\mathcal{E}, \mathcal{E}^{*}\right)$. Then $\Psi=K(T)$ satisfies (3.5). The identities constructed in such a way are of no physical significance and, therefore, they should be regarded as trivial. Notice that each trivial identity vanishes on shell. The converse implication is also true [3]: Each on-shell vanishing identity has the form $\Psi=K(T)$ for some skew-symmetric $K$. The proof exploits the regularity assumption for the equations of motion. The nontrivial identities are then defined as the elements of the quotient space $\operatorname{Id}(T)=\operatorname{Id}^{\prime}(T) / \operatorname{Id}_{0}(T)$, where $\operatorname{Id}_{0}(T) \subset \operatorname{Id}(T)$ is the subspace of trivial identities.

An identity $\Psi \in \operatorname{Id}(T)$ is called Noether's identity if there exists a vector bundle $\mathcal{G} \rightarrow$ $M$ together with a section $\xi \in \Gamma(\mathcal{G})$ and a homomorphism $Z \in \operatorname{Hom}\left(\mathcal{G}, \mathcal{E}^{*}\right)$ such that $\operatorname{Im} Z \subset \operatorname{Id}(T)$ and $\Psi=Z(\xi)$. Denote by $\operatorname{NId}(T) \subset \operatorname{Id}(T)$ the subspace of all Noether's identities and define the quotient space $\operatorname{Char}(T)=\operatorname{Id}(T) / \operatorname{NId}(T)$, whose elements are called characteristics. Again, one can choose the vector bundle $\mathcal{G}$ to be big enough so that $\operatorname{NId}(T)=\operatorname{Im} Z$. In this case, $Z$ is said to define an (over)complete basis of the generators of Noether's identities. Notice that $Z^{*}(T)=0$, where $Z^{*}$ is transpose of $Z$. It is quite natural to call $\mathcal{G}$ the bundle of the Noether identities. 
All we have said above can be concisely reformulated in terms of the following sequence of homomorphisms

$$
0 \longrightarrow \Gamma(\mathcal{F}) \stackrel{R}{\longrightarrow} \Gamma(T M) \stackrel{J}{\longrightarrow} \Gamma(\mathcal{E}) \stackrel{Z^{*}}{\longrightarrow} \Gamma\left(\mathcal{G}^{*}\right) \longrightarrow 0
$$

and its transpose

$$
0 \longleftarrow \Gamma\left(\mathcal{F}^{*}\right) \stackrel{R^{*}}{\longleftarrow} \Gamma\left(T^{*} M\right) \stackrel{J^{*}}{\longleftarrow} \Gamma\left(\mathcal{E}^{*}\right) \stackrel{Z}{\longleftarrow} \Gamma(\mathcal{G}) \longleftarrow 0 .
$$

Upon restriction to $\Sigma$ these sequences make cochain complexes; the properties $Z^{*} \circ J \approx 0$ and $J^{*} \circ Z \approx 0$ follow from the differential consequence of the identity $Z^{*}(T)=0$. The spaces of all rigid symmetries and characteristics are then naturally identified with the cohomology groups

$$
\operatorname{RSym}(T) \simeq \frac{\left.\operatorname{Ker} J\right|_{\Sigma}}{\left.\operatorname{Im} R\right|_{\Sigma}}, \quad \operatorname{Char}(T) \simeq \frac{\left.\operatorname{Ker} J^{*}\right|_{\Sigma}}{\left.\operatorname{Im} Z\right|_{\Sigma}} .
$$

Intimately related to the notion of an identity is the notion of a conservation law. The latter is identified with a form-valued differential operator $j \in \Omega^{n-1}$ taking true histories to closed $(n-1)$-forms,

$$
d j \approx 0
$$

In view of the regularity assumptions, the $n$-form $d j$ has to be proportional to the equations of motion, that is

$$
\int_{X} d j=\langle\Psi, T\rangle
$$

for some $\Psi \in \Gamma\left(\mathcal{E}^{*}\right)$. Comparing the last equality with (3.5), we see that every on-shell closed $(n-1)$-form gives rise to some (perhaps trivial) identity and vice versa. The relation

$$
\text { (Identities) } \leftrightarrow \text { (Conservation Laws) }
$$

is far from being a bijection, since neither left nor right hand sides of (3.11) are defined by $j$ and $\Psi$ unambiguously. For instance, one may add to $j$ any closed (and hence exact) form $d i$ without changing $\Psi$. The exact forms $d i \in \Omega^{n-1}$ are characterized by zero charge and, therefore, one should regard them as trivial. Another source of triviality is related to the currents $j$ that vanish on shell. Taking into account either of possibilities, we identify the space of nontrivial conservation laws $\mathrm{CL}(T)$ with the equivalence classes of on-shell closed forms $j \in \Omega^{n-1}$. Two such forms are considered as equivalent whenever they differ by an on-shell exact form?

$$
j \sim j^{\prime} \Leftrightarrow j-j^{\prime} \approx d i .
$$

\footnotetext{
${ }^{2}$ This definition does not exclude the possibility that the charge, being related to a nontrivial conserved current, vanishes identically. For discussion of this phenomenon, see [20].
} 
The space $\mathrm{CL}(T)$ is also known as the characteristic cohomology of $\Sigma$ in form degree $n-1$, see [18], [19] and [25].

Turning to the right hand side of (3.11), one can see that trivial identities give rise to trivial conservation laws. This is an additional (in fact the main) reason to call the on-shell vanishing identities trivial. Furthermore, the Noether identities correspond to the trivial conservation laws as well.

Considering (3.12) modulo trivialities, one arrives at the following relation

$$
\operatorname{Char}(T) \leftrightarrow \mathrm{CL}(T)
$$

This last relation is in essence an isomorphism [15], [5], so that the problem of constructing nontrivial conservation lows boils down to finding out characteristics.

Example. Generally it is a rather hard problem to identify the spaces of all non-trivial symmetries and characteristics for a given set of equations. The problem, however, is considerably simplified for the ordinary differential equations in normal form, where either of spaces admits a fairly explicit description. By this reason we will use this class of dynamical systems to exemplify all the general notions and constructions throughout the paper. Without loss of generality we can restrict ourselves to the systems of first-order ODEs

$$
T^{i} \equiv \dot{x}^{i}(t)+v^{i}(t, x)=0 .
$$

Here the overdot stands for the derivative with respect to the independent variable $t \in \mathbb{R}$ and $\left\{x^{i}\right\}$ are local coordinates on $Y$. Considering $Y \times \mathbb{R}$ as a trivial fiber bundle over $\mathbb{R}$, one can thought of $v$ as a vertical vector field on $Y \times \mathbb{R}$. Equations (3.15) are neither reducible nor gauge invariant and the dynamics bundle is naturally identified with the tangent bundle $T M$ of the trajectory space $M=\operatorname{Map}(\mathbb{R}, Y)$. By definition, the characteristics of (3.15) live in the space $\Gamma\left(T^{*} M\right)$ spanned by 1 -forms

$$
\Psi=\int d t \psi_{i}(t, x, \dot{x}, \ldots, \stackrel{(m)}{x}) \delta x^{i}(t) .
$$

The system (3.15) being regular, one can exclude all the derivatives from the integrand of (3.16) by means of the equations of motion, looking for characteristics of the form

$$
\Psi=\int d t \psi_{i}(t, x) \delta x^{i}(t)
$$

where $\psi=\psi_{i}(t, x) d x^{i}$ is a vertical 1-form on $Y \times \mathbb{R}$. Relation (3.5) results in two conditions

$$
\psi=\widetilde{d} f, \quad \partial_{t} f=v \cdot f
$$


where $f$ is a function on $Y \times \mathbb{R}$ and $\widetilde{d}$ is the exterior differential on $Y$. Let $C_{v}^{\infty}(Y \times$ $\mathbb{R})$ denote the space of all smooth functions satisfying equations (3.18). These equations specify $f$ up to an additive constant and each such $f$ is conserved. Thus we are led to the following isomorphisms:

$$
\operatorname{Char}(\dot{x}+v) \simeq C_{v}^{\infty}(Y \times \mathbb{R}) / \mathbb{R}, \quad \operatorname{CL}(\dot{x}+v) \simeq C_{v}^{\infty}(Y \times \mathbb{R})
$$

Let us now turn to the symmetries of (3.15). The general evolutionary vector field on $M$ has the form

$$
W=\int d t w^{i}(t, x, \dot{x}, \ldots, \stackrel{(m)}{x}) \frac{\delta}{\delta x^{i}(t)} .
$$

Being interested in symmetries, we can exclude all the derivatives from the integrand of (3.20) with the help of the equations of motion. This reduces the ansatz (3.20) to

$$
W=\int d t w^{i}(t, x) \frac{\delta}{\delta x^{i}(t)},
$$

where $w=w^{i}(t, x) \partial / \partial x^{i}$ is a vertical vector field on $Y \times \mathbb{R}$. Verifying (3.4) one can find that $W$ is a symmetry iff

$$
\partial_{t} w=[v, w] .
$$

Denoting by $\mathfrak{X}_{v}(Y \times \mathbb{R})$ the space of all vertical vector fields satisfying $(\underline{3.22})$, we can write

$$
\operatorname{Sym}(\dot{x}+v)=\mathfrak{X}_{v}(Y \times \mathbb{R}) .
$$

Let us suppose that the vector field $v$ is complete. Then, considering (3.18) and (3.22) as systems of the first-order ODEs for $f$ and $w$, respectively, we conclude that the spaces of conservation laws and symmetries are isomorphic to the spaces of initial data $C^{\infty}(Y)$ and $\mathfrak{X}(Y)$. Thus the space of symmetries appears to be "much larger" than the spaces of characteristics and conservation laws whenever $\operatorname{dim} Y>1$.

The form of equations (3.4) and (3.6) shows a certain duality between the concepts of symmetry and conservation law. The dual nature of these concepts is also supported by the celebrated Noether theorem for Lagrangian equations of motion. The long time use of this theorem has lead to a widespread belief that any conservation law or, what is the same, characteristic is a reflection of some symmetry. Beyond the scope of Lagrangian dynamics, this is not always true. Not any conservation law comes from some symmetry, nor does each symmetry correspond to a conservation law. There is no canonical relationship between these two concepts in general. This fact has a simple geometric explanation: The characteristics $\Psi$ 's and the symmetries $X$ 's belong to different vector bundles unless 
$\mathcal{E}^{*}=T M$. This suggests that (i) any systematic procedure for converting symmetries to characteristics and vice versa has to involve an additional geometric structure on $M$ and (ii) whatever this structure may be, it will result in a linear relation between the dual of the dynamics bundle $\mathcal{E}^{*}$ and the tangent bundle of $M$. It is then quite reasonable to start with some homomorphism $V: \mathcal{E}^{*} \rightarrow T M$ and to look for a suitable set of conditions ensuring that $V(\Psi)$ is a symmetry whenever $\Psi$ is a characteristic. Actually, such a homomorphism $V$ has been already identified as a key structure needed for the path-integral quantization of non-Lagrangian gauge theories [7]. This homomorphism is called a Lagrange anchor.

\section{The Lagrange anchor}

Definition 4.1. A vector bundle homomorphism $V: \mathcal{E}^{*} \rightarrow T M$ is called a Lagrange anchor, if the diagram

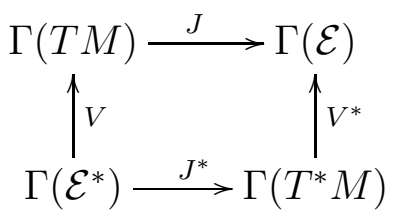

commutes upon restriction to the shell, i.e.,

$$
J \circ V \approx V^{*} \circ J^{*} .
$$

Since the last relation is linear in $V$, the Lagrange anchors form a vector space, which we denote by $\operatorname{An}^{\prime}(T)$. This space is clearly nonempty as each on-shell vanishing homomorphism $V$ obeys (4.2). The on-shell vanishing Lagrange anchors are of no significance from the perspective of converting conservation laws to rigid symmetries and we rule them out by passing to the quotient $\operatorname{An}(T)=\operatorname{An}^{\prime}(T) / \operatorname{An}_{0}(T)$, where $\operatorname{An}_{0}(T)$ is the subspace of all on-shell vanishing Lagrange anchors.

Proposition 4.1. The Lagrange anchor takes identities to symmetries, trivial identities to trivial symmetries and the Noether identities to the gauge symmetries.

Proof. If $\Psi$ is an identity, then $J^{*}(\Psi) \approx 0$ and

$$
J \circ V(\Psi) \approx V^{*} \circ J^{*}(\Psi) \approx 0 .
$$

Hence $X=V(\Psi)$ is a symmetry. It is also clear that $X \approx 0$ whenever $\Psi \approx 0$. If now $\Psi=Z(\xi)$ for some $\xi \in \Gamma(\mathcal{G})$, then

$$
J \circ V(\Psi) \approx V^{*} \circ J^{*} \circ Z(\xi) \approx 0 \quad \forall \xi \in \Gamma(\mathcal{G}) .
$$


Thus, $X=V \circ Z(\xi)$ is a gauge symmetry. Since the generators $R$ are assumed to form an (over)complete basis in the gauge algebra bundle, there exists a homomorphism $W: \mathcal{G} \rightarrow$ $\mathcal{F}$ such that the diagram

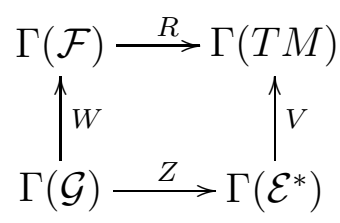

commutes upon restriction to the shell, i.e., $V \circ Z \approx R \circ W$.

Let us combine all the diagrams (3.7), (3.8), (4.1), (4.5) into the following one:

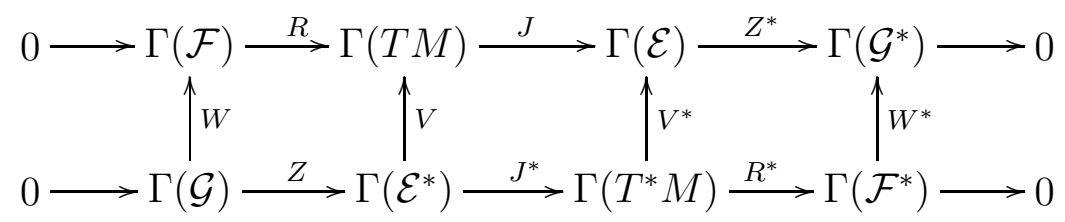

From the previous section we know that the rows of this diagram make cochain complexes upon restriction to the shell. Then, the on-shell commutativity of the squares implies that the upward arrows define a cochine map. It is the standard fact of homological algebra that each cochain map induces a well defined homomorphism in cohomology. In the case at hand this gives the homomorphism

$$
H(V): \operatorname{Char}(T) \rightarrow \operatorname{RSym}(T)
$$

from the space of characteristics to the space of rigid symmetries.

A natural question arises about identifying the different anchors from $\operatorname{An}(T)$ which induce the same homomorphism (4.7). An appropriate algebraic concept for examining the question is that of homotopy. We say that two anchors $V, \tilde{V} \in \operatorname{An}(T)$ are equivalent, $V \sim \tilde{V}$, if the corresponding cochain maps are homotopic. The latter implies the existence of homomorphisms $G$ and $K$ such that

$$
\begin{array}{ll}
\tilde{V}-V \approx G \circ J^{*}+R \circ K, & \tilde{V}^{*}-V^{*} \approx J \circ G+K^{*} \circ R^{*}, \\
\tilde{W}-W \approx K \circ Z, & \tilde{W}^{*}-W^{*} \approx Z^{*} \circ K^{*} .
\end{array}
$$

All the relevant maps are depicted in the following diagram:

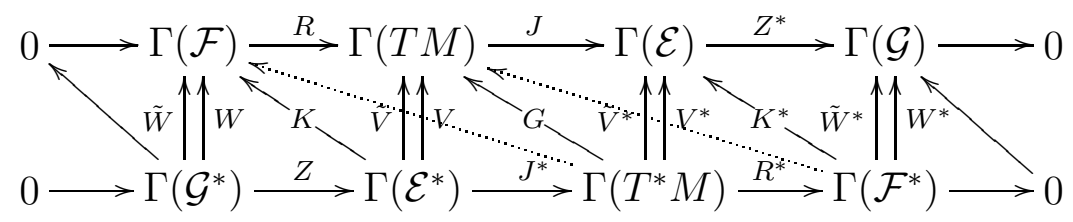


The weak equalities in the first line of 4.8 imply that

$$
J \circ\left(G-G^{*}\right) \approx 0 .
$$

This is the only condition on the homomorphisms $G$ and $K$. Taking into account the fact of completeness of the gauge algebra generators, we can rewrite (4.10) in the following equivalent form:

$$
G-G^{*} \approx R \circ U
$$

for some $U: T^{*} M \rightarrow \mathcal{F}$ satisfying

$$
R \circ U+U^{*} \circ R^{*} \approx 0 .
$$

In the diagram above, the homomorphisms $U$ and $-U^{*}$ are depicted by the dotted arrows, so that the corresponding parallelogram of maps appears to be on-shell commutative. Since the homotopic cochain maps are known to induce the same homomorphism in cohomology, we conclude that $H(V)=H(\tilde{V})$ whenever the anchors $V$ and $\tilde{V}$ are equivalent.

Denote by $\operatorname{HAn}(T)$ the space of the homotopy classes of Lagrange anchors. This can be thought of as the quotient of $\operatorname{An}^{\prime}(T)$ by the Lagrange anchors of the form

$$
V_{\text {triv }}=R \circ K+G \circ J^{*}+V_{0},
$$

where $V_{0} \approx 0$ and $G \approx G^{*}$. We have

$$
V_{\text {triv }}(\Psi)=R(\varepsilon)-X_{0}
$$

where $\varepsilon=K(\Psi)$ and $X_{0}=G \circ J^{*}(\Psi)+V_{0}(\Psi) \approx 0$. In other words, the trivial Lagrange anchor $V_{\text {triv }}$ takes each identity (trivial or not) to a trivial rigid symmetry.

Example (continuation). Consider the dynamical system (3.15). Then the most general local ansatz for the Lagrange anchor $V: T^{*} M \rightarrow T M$ looks like

$$
\langle V(W), P\rangle \doteq\left\langle W, V^{*}(P)\right\rangle=\sum_{n=0}^{N} \int d t w_{i}(t) \alpha_{n}^{i j}(t, x, \dot{x}, \ldots, \stackrel{(m)}{x}) \stackrel{(n)}{p}_{j}(t)
$$

where $W=w_{i} \delta x^{i}$ and $P=p_{i} \delta x^{i}$ are arbitrary 1-forms on $M$. The homomorphism $J: T M \rightarrow T M$ is given by the matrix first-order differential operator

$$
J=\left(\delta_{j}^{i} \frac{d}{d t}+\frac{\partial v^{i}}{\partial x^{j}}\right) \delta\left(t-t^{\prime}\right)
$$

\footnotetext{
${ }^{3}$ These equivalence relations for the Lagrange anchors originally appeared in [9]. As usual, the homotopy equivalence classes of cochain transformations can be equivalently reinterpreted as a cohomology classes of corresponding cochain complex [7]. In the context of local field theory the equivalence classes of Lagrange anchors were recently discussed in [21].
} 
The peculiar form of the operator $J$ allows one to absorb all the derivatives of $p$ 's into the trivial anchor (4.13) for an appropriate $G$. Using then the equations of motion or, what is the same, adding an appropriate on-shell vanishing anchor $V_{0}$, one can also exclude all the derivatives of $x$ 's from the integrand of (4.15). We are led to conclude that each equivalence class of the Lagrange anchors for equations (3.15) contains the anchor of the form

$$
V\left(\delta x^{i}(t)\right)=\alpha^{i j}(t, x) \frac{\delta}{\delta x^{j}} .
$$

Substituting the last expression to the defining condition (4.2), we finally get

$$
\alpha^{i j}=-\alpha^{j i}, \quad \partial_{t} \alpha=L_{v} \alpha,
$$

Thus, there is a one-to-one correspondence between the space of non-equivalent Lagrange anchors $\operatorname{An}(\dot{x}+v)$ and the vertical bivector fields $\alpha=\alpha^{i j}(t, x) \partial_{i} \wedge \partial_{j}$ on $Y \times \mathbb{R}$ satisfying (4.18). If the vector field $v$ is complete, then equation (4.18) defines an isomorphism $\operatorname{An}(\dot{x}+v) \simeq \Gamma\left(\wedge^{2} T Y\right)$. This classification result is in agreement with the conclusions of paper [21]. In [21], it is proven that any Lagrange anchor for the AKSZ-type sigma models [32] always reduces to a purely algebraic operator whose coefficients do not dependent on the space-time derivatives of fields.

\section{Proper symmetries and proper deformations}

We begin with an alternative geometric interpretation of the Lagrange anchor as a "Lie algebroid with relaxed integrability condition". It was first introduced in [7], and termed the Lagrange structure. To fix ideas, let us think for a while of $\mathcal{E} \rightarrow M$ as if it were an ordinary vector bundle over a finite dimensional manifold 4 . The way of adapting this construction to the context of local field theory will be obvious.

Definition 5.1. Given a classical system $(\mathcal{E}, T)$, a Lagrange structure is a homomorphism $\mathrm{d}_{\mathcal{E}}: \Gamma\left(\wedge^{\bullet} \mathcal{E}\right) \rightarrow \Gamma\left(\wedge^{\bullet+1} \mathcal{E}\right)$ obeying two conditions:

(i) $\mathrm{d}_{\mathcal{E}}$ is a derivation of degree 1, i.e.,

$$
\mathrm{d}_{\mathcal{E}}(A \wedge B)=\mathrm{d}_{\mathcal{E}} A \wedge B+(-1)^{p} A \wedge \mathrm{d}_{\mathcal{E}} B
$$

for any $A \in \Gamma\left(\wedge^{p} \mathcal{E}\right)$ and $B \in \Gamma\left(\wedge^{\bullet} \mathcal{E}\right)$;

\footnotetext{
${ }^{4}$ See comments at the end of Sec. 2 .
} 
(ii) $\mathrm{d}_{\mathcal{E}} T=0$.

Here we identify $\Gamma\left(\wedge^{0} \mathcal{E}\right)$ with $C^{\infty}(M)$.

Due to the Leibnitz rule (i), in each trivializing chart $U \subset M$ the operator $\mathrm{d}_{\mathcal{E}}$ is completely determined by its action on the coordinate functions $\varphi^{i}$ and the frame sections $\left\{e^{a}\right\}$ of $\left.\mathcal{E}\right|_{U}$ :

$$
\mathrm{d}_{\mathcal{E}} \varphi^{i}=V_{a}^{i}(\varphi) e^{a}, \quad \mathrm{~d}_{\mathcal{E}} e^{a}=\frac{1}{2} C_{b c}^{a}(\varphi) e^{b} \wedge e^{c} .
$$

Applying $\mathrm{d}_{\mathcal{E}}$ to the section $T=T_{a} e^{a}$, one can see that the property (ii) is equivalent to the structure relations

$$
\mathrm{d}_{\mathcal{E}} T=\frac{1}{2}\left(V_{a}^{i} \partial_{i} T_{b}-V_{b}^{i} \partial_{i} T_{a}+C_{a b}^{c} T_{c}\right) e^{a} \wedge e^{b}=0 .
$$

Clearly, the derivation $\mathrm{d}_{\mathcal{E}}$ defines a bundle homomorphism $V: \mathcal{E}^{*} \rightarrow T M$. The shell $\Sigma$ being a regularly imbedded submanifold, equation (5.2) is equivalent to the defining condition (4.2). Thus, $V$ is a Lagrange anchor.

Dualizing relations (5.1) one gets a bracket

$$
[\cdot, \cdot]: \Gamma\left(\mathcal{E}^{*}\right) \wedge \Gamma\left(\mathcal{E}^{*}\right) \rightarrow \Gamma\left(\mathcal{E}^{*}\right)
$$

On frame sections $e_{a}$ of $\left.\mathcal{E}^{*}\right|_{U}$ it reads

$$
\left[e_{a}, e_{b}\right]=C_{a b}^{d} e_{d}
$$

and extends to arbitrary $\Psi, \Psi^{\prime} \in \Gamma\left(\mathcal{E}^{*}\right)$ by the Leibnitz rule

$$
\left[f \Psi, \Psi^{\prime}\right]=f\left[\Psi, \Psi^{\prime}\right]+(V(\Psi) \cdot f) \Psi^{\prime}, \quad \forall f \in C^{\infty}(M)
$$

Generally the bracket violates the Jacobi identity, therefore it is not a Lie-algebra bracket.

Definition 5.2. A Lagrange structure $\left(\mathcal{E}, T, \mathrm{~d}_{\mathcal{E}}\right)$ is said to be integrable if $\mathrm{d}_{\mathcal{E}}^{2}=0$.

Comparing Definitions 5.1 and 5.2 with the definition of a Lie algebroid (see e.g. [22], [23]), one can see that the integrable Lagrange structure is nothing else but the Lie algebroid over $M$ with anchor $V: \mathcal{E}^{*} \rightarrow T M$, Lie bracket (5.3), and a distinguished section $T$. This particular yet important case accounts for the name of $V$ - "Lagrange anchor". For an integrable Lagrange structure the bracket (5.3) satisfies the Jacobi identity and the anchor distribution $\operatorname{Im} V \subset T M$ is integrable. 
To illustrate (as well as motivate) the definitions above let us consider a Lagrangian theory with action $S(\varphi)$. The equations of motion read

$$
T \equiv \mathrm{d} S=0
$$

so that the dynamics bundle $\mathcal{E}$ is given by the cotangent bundle $T^{*} M$ of the space of all histories. The canonical Lagrange structure is given by the exterior differential $\mathrm{d}$ : $\Gamma\left(\wedge^{\bullet} T^{*} M\right) \rightarrow \Gamma\left(\wedge^{\bullet+1} T^{*} M\right)$ and the defining condition (5.2) takes the form

$$
\mathrm{d} T=\mathrm{d}^{2} S=0 .
$$

The identity $\mathrm{d}^{2}=0$ means integrability. The Lagrange anchor is given by the identical map $V=$ id : $T M \rightarrow T M$ and the Lie bracket (5.3) coincides with the commutator of vector fields.

Definitions 5.1 and 5.2 suggest to view the Lagrange structure as a "Lie algebroid with relaxed integrability condition" in the sense that the "strong" integrability condition $\mathrm{d}_{\mathcal{E}}^{2}=0$ is replaced here by the weaker one $\mathrm{d}_{\mathcal{E}}^{2} T=0$. In spite of weakened integrability, it is still possible to utilize many of the usual differential-geometric constructions associated to Lie algebroids. In particular, we can endow the exterior algebra of $\mathcal{E}$-differential forms $\Gamma\left(\wedge^{\bullet} \mathcal{E}\right)$ with the operations of inner differentiation and Lie derivative.

By definition, the inner differential $i_{\Psi}$ associated with a section $\Psi \in \Gamma\left(\mathcal{E}^{*}\right)$ is a differentiation of $\Gamma\left(\wedge^{\bullet \mathcal{E}}\right)$ of degree -1 which action on $A \in \Gamma(\mathcal{E})$ is given by

$$
i_{\Psi} A=\langle\Psi, A\rangle
$$

Following the analogy with Cartan's calculus, the Lie derivative along $\Psi$ is defined now by

$$
L_{\Psi}=i_{\Psi} \circ \mathrm{d}_{\mathcal{E}}+\mathrm{d}_{\mathcal{E}} \circ i_{\Psi}
$$

It is a differentiation of $\Gamma\left(\wedge^{\bullet} \mathcal{E}\right)$ of degree 0 . One can easily verify the following identities:

$$
\begin{array}{ll}
\left\{i_{\Psi}, i_{\Psi^{\prime}}\right\}=0, & {\left[L_{\Psi}, \mathrm{d}_{\mathcal{E}}\right]=\left[i_{\Psi}, \mathrm{d}_{\mathcal{E}}^{2}\right],} \\
{\left[L_{\Psi}, i_{\Psi^{\prime}}\right]=i_{\left[\Psi, \Psi^{\prime}\right]},} & {\left[L_{\Psi}, L_{\Psi^{\prime}}\right]=L_{\left[\Psi, \Psi^{\prime}\right]}+\left\{i_{\Psi^{\prime}},\left[i_{\Psi}, \mathrm{d}_{\mathcal{E}}^{2}\right]\right\},}
\end{array}
$$

where the braces stand for anticommutators. We can also define the action of the Lie derivative on the contravariant $\mathcal{E}$-tensors by setting

$$
L_{\Psi} \Psi^{\prime}=\left[\Psi, \Psi^{\prime}\right] \quad \forall \Psi, \Psi^{\prime} \in \Gamma\left(\mathcal{E}^{*}\right) .
$$

This definition extends to arbitrary $\mathcal{E}$-tensor fields by the usual formulas of differential geometry. From this point on we return to infinite-dimensional setting of Sec. 2. 
Definition 5.3. Given a Lagrange structure $\left(\mathcal{E}, T, \mathrm{~d}_{\mathcal{E}}\right)$ and a section $\Psi \in \Gamma\left(\mathcal{E}^{*}\right)$, the vector field $V(\Psi)$ is said to be a proper symmetry generated by $\Psi$ if $L_{\Psi} T=0$.

Notice that the proper symmetry $V(\Psi)$ is a symmetry in the usual sense. Indeed, $L_{\Psi} T \approx J \circ V(\Psi)$ and $L_{\Psi} T=0$ implies $V(\Psi) \in \operatorname{Sym}^{\prime}(T)$. For variational equations of motion equipped with the canonical Lagrange structure (5.7) the proper symmetries coincide with the symmetries of action.

We emphasize that the property of being proper symmetry is not homotopy invariant: If $V(\Psi)$ is a proper symmetry and $V \sim V^{\prime}$, then $V^{\prime}(\Psi)$ may not be a symmetry at all, unless $\Psi \in \operatorname{Id}^{\prime}(T)$.

The image $\operatorname{Im} V \subset T M$ of the Lagrange anchor defines a generalized distribution on $M$ in the sense of Sussmann [24]. Recall that a generalized distribution $\mathcal{V}$ is said to be involutive, if it is closed under the Lie bracket, that is $[\mathcal{V}, \mathcal{V}] \subset \mathcal{V}$. The involutive closure of $\mathcal{V}$ is, by definition, the minimal involutive distribution $\overline{\mathcal{V}}$ containing $\mathcal{V}$ as a subdistribution. Clearly, $\overline{\mathcal{V}}$ is spanned by the iterated commutators of $\mathcal{V}$ and the equality $\mathcal{V}=\overline{\mathcal{V}}$ amounts to involutivity of $\mathcal{V}$.

Definition 5.4. A Lagrange anchor $V: \mathcal{E}^{*} \rightarrow T M$ is said to be transitive if $\operatorname{Im} V=T M$ and weakly transitive if $\overline{\operatorname{Im} V}=T M$.

For example, the canonical Lagrange anchor $V=\mathrm{id}: T M \rightarrow T M$ for Lagrangian equations of motion $T=\mathrm{d} S=0$ is both integrable and transitive.

Proposition 5.1. The Lagrange anchor $V: \mathcal{E}^{*} \rightarrow T M$ takes identities to proper symmetries. If the Lagrange anchor is weakly transitive, then each proper symmetry comes from some identity.

Proof. The first statement follows from the identity

$$
L_{\Psi} T=\mathrm{d}_{\mathcal{E}}\left(i_{\Psi} T\right)+i_{\Psi}\left(\mathrm{d}_{\mathcal{E}} T\right)=0 \quad \forall \Psi \in \operatorname{Id}^{\prime}(T)
$$

This identity also implies that

$$
\mathrm{d}_{\mathcal{E}}\left(i_{\Psi} T\right)=0
$$

for any generator of proper symmetry $\Psi$. If the function $i_{\Psi} T$ is annihilated by the anchor distribution $\operatorname{Im} V$, then it has to be annihilated by the involutive closure $\overline{\operatorname{Im} V}$. Since for transitive Lagrange anchors $\overline{\operatorname{Im} V}=T M$, we have $\mathrm{d}\left(i_{\Psi} T\right)=0$. Thus, $\langle\Psi, T\rangle \doteq 0$ and $\Psi \in \operatorname{Id}^{\prime}(T)$. 
Let $\operatorname{PSym}_{V}^{\prime}(T) \subset \operatorname{Sym}(T)$ denote the subspace of all proper symmetries and let $\operatorname{PSym}_{V}(T)$ be the quotient space of all proper symmetries by the trivial and gauge symmetries. According to this definition $\operatorname{PSym}_{V}(T)$ is a subspace in $\operatorname{RSym}(T)$. Combining Proposition 5.1 with Proposition 4.1, we arrive at the following statement.

Proposition 5.2. The bundle map $V: \mathcal{E}^{*} \rightarrow T M$ induces a well defined homomorphism

$$
H(V): \operatorname{Char}(T) \rightarrow \operatorname{PSym}_{V}(T)
$$

If the equivalence class $H(V)$ contains a weakly transitive Lagrange anchor, then the homomorphism (5.14) is surjective.

The symmetries of a classical system form the Lie algebra with respect to the vectorfield commutator. On the other hand, given a Lagrange anchor, the dual of the dynamics bundle carries the bracket operation (5.3). Having in mind the Lagrangian case, where characteristics coincide with the symmetries of action, one may expect a certain relationship between the aforementioned multiplicative structures in $\Gamma(T M)$ and $\Gamma\left(\mathcal{E}^{*}\right)$. This is detailed in the following proposition.

Proposition 5.3. If $V(\Psi)$ and $V\left(\Psi^{\prime}\right)$ are two proper symmetries, then $V\left(\left[\Psi, \Psi^{\prime}\right]\right)$ is also a proper symmetry. Furthermore, the subspace of identities $\operatorname{Id}^{\prime}(T) \subset \Gamma\left(\mathcal{E}^{*}\right)$ is closed with respect to the bracket (5.3), the trivial and Noether identities forming an ideal in $\operatorname{Id}^{\prime}(T)$. If the Lagrange structure is integrable, then $V: \Gamma\left(\mathcal{E}^{*}\right) \rightarrow \Gamma(T M)$ is a Lie algebra homomorphism and the bracket (5.3) makes $\operatorname{Id}^{\prime}(T)$ and $\operatorname{Char}(T)$ into the Lie algebras. In the latter case, we have a well defined Lie algebra homomorphism (5.14).

Proof. Applying the fourth identity (5.10) to $T$ and using (5.13), we find that $L_{\left[\Psi, \Psi^{\prime}\right]} T=0$. Hence $\left[\Psi, \Psi^{\prime}\right] \in \Gamma\left(\mathcal{E}^{*}\right)$ is a proper symmetry generator.

Applying now the third identity (5.10) to $T$, we get $i_{\left[\Psi, \Psi^{\prime}\right]} T=0$ provided that $\Psi, \Psi^{\prime} \in$ $\operatorname{Id}^{\prime}(T)$. So the bracket of two identities is an identity again. Suppose for a moment that $\Psi^{\prime}$ is given by a sum of the Noether and trivial identities, i.e., $\Psi^{\prime}=Z(\xi)+K(T)$, for some $K \in \Gamma\left(\mathcal{E}^{*} \wedge \mathcal{E}^{*}\right)$ and $\xi \in \Gamma(\mathcal{G})$. We have

$$
\begin{aligned}
{\left[\Psi, \Psi^{\prime}\right] } & =L_{\Psi} K(T)+L_{\Psi} Z(\xi) \\
& =\left(L_{\Psi} K\right)(T)+Z(V(\Psi) \xi)+\left(L_{\Psi} Z\right)(\xi)
\end{aligned}
$$

By definition, the right hand side is an identity. Clearly, the first term is a trivial identity and the second one is a Noether identity. Then the third term, being proportional to an arbitrary section $\xi$, has to define a Noether identity as well. We thus conclude that the trivial and 
Noether identities constitute an ideal in $\operatorname{Id}^{\prime}(T)$ with respect to the bracket multiplication. So we have a well defined bracket on the quotient space Char $(T)$.

It follows from the fourth identity in (5.10) that

$$
\left[V(\Psi), V\left(\Psi^{\prime}\right)\right] \cdot f-V\left(\left[\Psi, \Psi^{\prime}\right]\right) \cdot f=W\left(\Psi, \Psi^{\prime}\right) \cdot f \quad \forall f \in C^{\infty}(M)
$$

where

$$
W\left(\Psi, \Psi^{\prime}\right) \cdot f \equiv\left\langle\Psi \wedge \Psi^{\prime}, \mathrm{d}_{\mathcal{E}}^{2} f\right\rangle .
$$

By construction, the vector field $W\left(\Psi, \Psi^{\prime}\right)$ is a symmetry, not necessary proper though. It vanishes for integrable Lagrange structures, when the bracket (5.3) enjoys the Jacobi identity. In this case, relation (5.16) says that $V: \Gamma\left(\mathcal{E}^{*}\right) \rightarrow \Gamma(T M)$ is a Lie algebra homomorphism. This homomorphism takes the subalgebra $\operatorname{Id}^{\prime}(T)$ to the subalgebra $\operatorname{PSym}_{V}^{\prime}(T)$. Passing to the quotient, we get a well defined Lie algebra homomorphism (5.14).

Example (continuation). Besides the homomorphism $V: T^{*} M \rightarrow T M$, the Lagrange anchor (4.17) defines a bracket (5.3) on the cotangent bundle $T^{*} M$. The latter is given by

$$
\left[\delta x^{i}(t), \delta x^{j}\left(t^{\prime}\right)\right]=\delta x^{k}(t) \partial_{k} \alpha^{i j}(t, x(t)) \delta\left(t-t^{\prime}\right) \text {. }
$$

Formulas (4.17), (4.18) and (5.18) taken together define the most general Lagrange structure associated to the first-order differential equations (3.15).

Clearly, the Lagrange anchor (4.17) is transitive if for any $t \in \mathbb{R}$ the bivector $\alpha_{t} \in$ $\Gamma\left(\wedge^{2} T Y\right)$ is nondegenerate. In this case, each fiber $\left(Y, \alpha_{t}\right)$ of $Y \times \mathbb{R}$ is an almost symplectic manifold. One can also check [7] that the integrability of the Lagrange anchor (4.17) amounts to the Jacobi identity

$$
\frac{1}{6}[\alpha, \alpha] \equiv \alpha^{i m} \partial_{m} \alpha^{j k} \partial_{i} \wedge \partial_{j} \wedge \partial_{k}=0
$$

Here the square brackets denote the Schouten commutator of polyvector fields. In the integrable case, the bundle map $\alpha_{t}: T^{*} Y \rightarrow T Y$ defines the Lie algebroid of the Poisson manifold $\left(Y, \alpha_{t}\right)$. If the Lagrange anchor (4.17) is both transitive and integrable, then $\left(Y, \alpha_{t}\right)$ is a symplectic manifold.

Suppose now that for any $t \in \mathbb{R}$ the involutive closure of the vector distribution $\operatorname{Im} \alpha_{t} \subset$ $T Y$ coincides with the whole tangent bundle $T M$. Then the Lagrange anchor (4.17), (4.18) is weakly transitive. The condition for the 1 -form (3.17) to be a proper symmetry of (3.15) gives

$$
i_{X}(\widetilde{d} \psi)=0, \quad i_{X}\left(\partial_{t} \psi+\widetilde{d} \psi(v)\right)=0
$$


for any vertical vector field $X=\alpha(w)$, with $w$ being a 1-form. It follows from the first equation in (5.20) that

$$
L_{X} \widetilde{d} \psi=0 \quad \Rightarrow \quad \widetilde{d} \psi=0,
$$

since the vector fields $X=\alpha_{t}(w)$ generate the whole Lie algebra $\mathfrak{X}(Y)$. If the first group of the De Rham cohomology of $Y$ is trivial, then $\psi=\widetilde{d} f^{\prime}$ for some function $f^{\prime} \in C^{\infty}(Y \times \mathbb{R})$ and the second equation in (5.20) takes the form

$$
X \cdot\left(\partial_{t} f^{\prime}+v \cdot f^{\prime}\right)=0
$$

Again, the transitivity implies that $\partial_{t} f^{\prime}+v \cdot f^{\prime}=\dot{g}(t)$ for some $g \in C^{\infty}(\mathbb{R})$. Setting $f=f^{\prime}-g$, one can see that the 1 -form $\psi=\widetilde{d} f$ defines a characteristic for (3.15) in accordance with (3.18). So, every proper symmetry for the transitive Lagrange anchor (4.17) comes from some characteristic. This is in agreement with Proposition 5.2, In the case being considered the homomorphism (5.14) is actually an isomorphism. Indeed, if $X$ is the proper symmetry corresponding to a characteristic $\Psi=\mathrm{d} \int f(t, x) d t$, then $X=0$ implies that $\alpha(w) \cdot f=0$ for any 1 -form $w$. In view of the weak transitivity, $f$ has to be a function of $t$ alone. Then, $\Psi=0$. We thus arrive at the following generalization of the Noether theorem for the first-order ODEs:

Let $V$ be the weakly transitive Lagrange anchor (4.17) associated to the differential equations (3.15) and a vertical bivector field $\alpha$ on $Y \times \mathbb{R}$ with $H^{1}(Y)=0$, then

$$
\operatorname{Char}(\dot{x}+v) \simeq \operatorname{PSym}_{\alpha}(\dot{x}+v) .
$$

Consider now an integrable Lagrange structure $\left(\mathcal{E}, T, \mathrm{~d}_{\mathcal{E}}\right)$. In the integrable case, the dual of the dynamics bundle $\mathcal{E}^{*} \rightarrow M$ carries the structure of Lie algebroid and the differential $\mathrm{d}_{\mathcal{E}}: \Gamma\left(\wedge^{\bullet} \mathcal{E}\right) \rightarrow \Gamma\left(\wedge^{\bullet+1} \mathcal{E}\right)$ makes the exterior algebra of $\mathcal{E}$-differential forms into a cochain complex. Let $H^{\bullet}(\mathcal{E})=\operatorname{Kerd}_{\mathcal{E}} / \operatorname{Imd}_{\mathcal{E}}$ denote the corresponding cohomology groups. Classical dynamics on $M$ are specified by a 1-cocycle $T$. If $T^{\prime}$ is another representative of the $\mathrm{d}_{\mathcal{E}}$-cohomology class $[T] \in H^{1}(\mathcal{E})$, then

$$
T^{\prime}=T+\mathrm{d}_{\mathcal{E}} S
$$

for some $S \in C^{\infty}(M)$. We refer to the last relation by saying that the equations $T^{\prime}=0$ are obtained by a proper deformation of the equations $T=0$; the function $S$ is called a twist. The relevance of this notion to the context of conservation laws and proper symmetries is evident from the following simple observation. If $S$ is invariant with respect to the characteristic $\Psi \in \operatorname{Char}(T)$,

$$
L_{\Psi} S \doteq 0
$$


then $\Psi \in \operatorname{Char}\left(T^{\prime}\right)$. Indeed,

$$
i_{\Psi} T^{\prime}=i_{\Psi}\left(T+\mathrm{d}_{\mathcal{E}} S\right) \doteq 0 .
$$

Notice that the conserved current of the deformed equations $T^{\prime}$ can be different from that of the equations $T$, even though the characteristic is the same.

In general the proper deformation (5.24) can be quite nontrivial and by no means reduces to equivalence transformations of the original equations $T$, like taking linear combinations of the equations or change of variables. To exemplify the non-triviality of the deformation (5.24), it is sufficient to consider a proper deformations of the simplest possible differential equations

$$
T^{i} \equiv \dot{x}^{i}(t)=0 \text {. }
$$

As is known, these equations enjoy an integrable Lagrange structure given by a vertical Poisson bivector $\alpha=\alpha^{i j}(t, x) \partial_{i} \wedge \partial_{j}$ on $Y \times \mathbb{R}$. Taking the twist $S$ in the form

$$
S=-\int d t H(t, x(t))
$$

where $H(t, x)$ is an arbitrary function, we get the Hamiltonian equations

$$
T^{\prime i}=T^{i}-\alpha^{i j} \frac{\delta S}{\delta x^{j}}=\dot{x}^{i}-\left\{x^{i}, H\right\}=0 .
$$

So, the proper deformation is quite nontrivial as it can transform the Hamiltonian equations with a given Hamiltonian into equations with any other Hamiltonian and the same Poisson bracket. The characteristics for the original equations (5.27) have the form $\Psi=$ $\mathrm{d} \int f(x(t)) d t$, where $f(x)$ is an arbitrary function. If the twist (5.28) is invariant with respect to $\Psi$, then $\{f, H\}=\dot{g}(t)$ and the value $f-g$ is an integral of motion of (5.29).

\section{Field-theoretical examples}

To further illustrate the concept of proper symmetry we consider here some well-known examples of non-Lagrangian field equations. In addition, we will see what the Lagrange anchor looks like in local field theory.

\section{1 $p$-form fields}

In this subsection, we consider the theory of free $p$-form fields in the strength-tensor formalism. We will show that the notion of Lagrange anchor enables one to relate all the 
space-time symmetries with conserved currents expressible through the energy-momentum tensor. In the Lagrangian framework, relied on the notion of gauge potential, this relationship is just an immediate consequence of the Noether theorem. Our consideration, however, does not suppose the existence of any action functional.

Given an $n$-dimensional Riemannian manifold $(X, g)$, consider a $p$-form field $F \in$ $\Lambda^{p}(X)$ subject to equations

$$
T_{1}(F) \equiv d F=0, \quad T_{2}(F) \equiv d * F=0
$$

Here $*: \Lambda^{p}(X) \rightarrow \Lambda^{n-p}(X)$ is the Hodge operator associated to the metric $g$. The equations enjoy no gauge symmetry obeying the Noether identities

$$
d T_{1}=0, \quad d T_{2}=0
$$

The last fact points up the non-Lagrangian nature of the field equations 6.15

To make contact with the general definitions of the previous sections let us mention that the configuration space of fields $M$ is given here by the space $\Lambda^{p}(X)$ and the sections of the dynamics bundle, in particular the equations of motion $T=\left(T_{1}, T_{2}\right)$, take values in the space $\Lambda^{p+1}(X) \oplus \Lambda^{n-p+1}(X)$. Since $M$ is a linear space, we can identify the tangent space $T_{F} M$ at a point $F \in M$ with the space $M=\Lambda^{p}(X)$ itself. Furthermore, by making use of the standard inner product on $\Lambda(X)$,

$$
(A, B)=\int_{X} A \wedge * B
$$

we identify the vector spaces $\Lambda^{p}(X)$ and $\Lambda^{p+1}(X) \oplus \Lambda^{n-p+1}(X)$ with their dual spaces.

One can easily check that the field equations (6.1) admit the two-parameter family of Lagrange anchors $V$ defined by

$$
\langle V(W), P\rangle \doteq\left\langle W, V^{*}(P)\right\rangle=a\left(W_{1}, d P\right)+b\left(W_{2}, d * P\right)
$$

for all $W=\left(W_{1}, W_{2}\right) \in \Lambda^{p+1}(X) \oplus \Lambda^{n-p+1}(X), P \in \Lambda^{p}(X)$, and $a, b \in \mathbb{R}$. This family is a straightforward generalization of the Lagrange anchor proposed in [7] for Maxwell's electrodynamics in the strength-tensor formalism.

Since the equations of motion (6.1) are linear and the anchor (6.4) does not depend on fields, the bracket (5.3) is zero and the corresponding Lagrange structure appears to be

\footnotetext{
${ }^{5}$ Of course, one can solve the first equation 6.1 in terms of a gauge potential, $F=d A$, following which the second equation (6.1) becomes Lagrangian, but we are interested in treating these equations as they are, i.e., without passing to any equivalent Lagrangian formulation.
} 
integrable. To investigate its transitivity we should consider the kernel of the operator $V^{*}$. By definition, a $p$-form $P$ belongs to ker $V^{*}$ if

$$
\left\langle W, V^{*}(P)\right\rangle=0 \quad \forall W .
$$

Suppose that $a b \neq 0$, then the last condition is equivalent to the equations

$$
d P=0, \quad d * P=0
$$

which coincide in form with the equations of motion (6.1) for the field $F$. Since equations (6.6) enjoy no gauge symmetry, their general solution $P$ cannot depend on arbitrary functional parameters 6 . In particular, it cannot depend on the field $F$. We refer to this situation by saying that the Lagrange anchor (6.4) is almost transitive for $a b \neq 0$.

Notice also that the Lagrange anchor (6.4) is nontrivial whenever $a \neq b$. In case $a=b$, we can write 6.4 as

$$
\left(W_{1}, T_{1}(a P)\right)+\left(W_{2}, T_{2}(a P)\right) .
$$

The last expression corresponds to the trivial Lagrange anchor (4.13) with $G(P)=a P$.

Recall that a vector field $\xi \in \mathfrak{X}(X)$ is called a Killing vector of the metric $g$ if $L_{\xi} g=0$. Each Killing vector gives rise to both the symmetry transformation

$$
\delta_{\xi} F=L_{\xi} F
$$

of the field equations (6.1) and the characteristic $\Psi=\left(\Psi_{1}, \Psi_{2}\right) \in \Lambda^{p+1}(X) \oplus \Lambda^{n-p+1}(X)$. The latter is given by

$$
\Psi_{1}=(-1)^{(n-p)(p-1)} * i_{\xi} * F, \quad \Psi_{2}=(-1)^{p-1} * i_{\xi} F
$$

It is easy to verify that

$$
\langle\Psi, T\rangle=\left(\Psi_{1}, T_{1}\right)+\left(\Psi_{2}, T_{2}\right)=\int_{X} d j
$$

where the on-shell closed $(n-1)$-form $j$ reads

$$
j=\frac{1}{2}\left(\left(i_{\xi} F\right) \wedge * F+(-1)^{p-1} F \wedge\left(i_{\xi} * F\right)\right) .
$$

In terms of local coordinates $\left\{x^{\mu}\right\}$ on $X$ the corresponding conserved current is given by the 1-form

$$
* j=\xi^{\mu} T_{\mu \nu}(x) d x^{\nu},
$$

\footnotetext{
${ }^{6}$ For instance, if $X$ is a compact manifold without boundary, then the solutions to Eqs. (6.6) are the harmonic $p$-forms on $X$. These form a finite dimensional vector space isomorphic to $H^{p}(X)$.
} 
$T_{\mu \nu}=T_{\nu \mu}$ being the symmetric energy-momentum tensor of the $p$-form field $F$.

In view of Proposition 5.1, the characteristic $\Psi$ is bound to be the generator of a proper symmetry. A simple calculation gives

$$
\delta_{\xi} F=V(\Psi) \approx(a-b) L_{\xi} F
$$

So, up to an overall constant, which can be included in $\xi$, the transformation (6.13) coincides with the Lie derivative of the $p$-form $F$ along the Killing vector $\xi$. As would be expected, the transformation becomes trivial when $a=b$ (the case of trivial anchor (6.7)).

To summarize, each infinitesimal isometry of the Riemannian manifold $(X, g)$ gives rise to a conservation law with the current (6.12). The Lagrange anchor (6.4) allows us to consider this conservation law as coming from the proper symmetry (6.13) of the field equations (6.1). Moreover, as the Lagrange anchor is almost transitive for $a b \neq 0$, one can be sure that each proper symmetry $V(\Psi)$ results in a conservation law provided that the generator $\Psi$ depends on $F$.

All the above formulas hold true for conformal Killing vectors $\xi$ in the critical dimension $n=2 p$. This follows from the conformal invariance of the Hodge operator $*: \Lambda^{p}(X) \rightarrow \Lambda^{p}(X)$.

\subsection{Self-dual $p$-form fields}

Let $(X, g)$ be a $(4 k+2)$-dimensional pseudo-Riemannian manifold of Lorentz signature. Then the Hodge operator $*: \Lambda^{2 k+1}(X) \rightarrow \Lambda^{2 k+1}(X)$ squares to +1 on the middle forms and we have the direct sum decomposition $\Lambda^{2 k+1}(X)=\Lambda_{+}^{2 k+1}(X) \oplus \Lambda_{-}^{2 k+1}(X)$, where $\Lambda_{ \pm}^{2 k+1}(X)$ are the subspaces of (anti-)self-dual $(2 k+1)$-forms on $X$. The subspaces $\Lambda_{ \pm}^{2 k+1}(X)$ are known to be isotropic with respect to the standard inner product 6.3 . Therefore, the inner product defines a non-degenerate pairing between the spaces $\Lambda_{+}^{2 k+1}(X)$ and $\Lambda_{-}^{2 k+1}(X)$, so that we can regard these spaces as being dual to each other.

Consider now a self-dual $(2 k+1)$-form field $H^{+}$subject to the closedness condition

$$
T\left(H^{+}\right) \equiv d H^{+}=0
$$

Regarding this condition as equations of motion, we see that the sections of the corresponding dynamics bundle $\mathcal{E} \rightarrow M$ take values in $\Lambda^{2 k+2}(X)$ and $M$ is given by $\Lambda_{+}^{2 k+1}(X)$. The tangent space to each field configuration $H^{+} \in M$ can be identified with $M$ as before. Equations (6.14) are known to be non-Lagrangian unless one introduces auxiliary fields 
or breaks the manifest covariance [26, 27, 28, 29, 30, 31]. There is the Noether identity $d T=0$ but no gauge symmetry. It was shown in [11] that the theory (6.14) admits a natural Lagrange structure defined by the anchor

$$
\langle V(W), P\rangle \doteq\left\langle W, V^{*}(P)\right\rangle=(W, d P)
$$

where $W \in \Lambda^{2 k+2}(X)$ and $P \in \Lambda_{-}^{2 k+1}(X)$. Again, the Lagrange anchor is almost transitive, since the condition $d P=0$ implies that the anti-self-dual form $P$ does not depend on the field $H^{+}$.

If $\xi \in \mathfrak{X}(X)$ is a conformal Killing vector of the metric $g$, then $\Psi=-* i_{\xi} H^{+} \in$ $\Lambda^{2 k+2}(X)$ is a characteristic for (6.14). Indeed, using the identity $H^{+} \wedge L_{\xi} H^{+}=0$, one can readily find

$$
\langle\Psi, T\rangle=-\left(* i_{\xi} H^{+}, d H^{+}\right)=\int_{X} d j, \quad j=\frac{1}{2} i_{\xi} H^{+} \wedge H^{+} .
$$

By analogy with the previous case we can define the energy-momentum tensor $T_{\mu \nu}$ of the self-dual field $H^{+}$through the conserved current $* j=\xi^{\mu} T_{\mu \nu}(x) d x^{\nu}$. It is easy to see that the tensor $T_{\mu \nu}$ is symmetric and traceless.

According to Proposition 5.1, the anchor (6.15) takes the characteristic $\Psi$ to a proper symmetry of the equations of motion (6.14). As one would expect, the corresponding variation of $\mathrm{H}^{+}$is given by the Lie derivative along the conformal Killing vector modulo on-shell vanishing terms:

$$
\delta_{\xi} H^{+}=V(\Psi) \approx L_{\xi} H^{+}
$$

Conversely, one could start with the proper symmetry transformation 6.17), which presence is evident from the very definition of the theory (6.14), and then argue that the generator $\Psi$ is nothing else but a characteristic to the field equations since the Lagrange anchor (6.15) is almost transitive. This gives the desired relation between the space-time symmetries of the model and the conservation laws.

\subsection{Chiral bosons in two dimensions}

Consider now a multiplet of $N$ self-dual 1 -forms $H_{a}^{+}, a=1, \ldots, N$, in two dimensional Minkowski space $\mathbb{R}^{1,1}$. As above, the field equations have the form of closedness condition

$$
T_{a}\left(H^{+}\right) \equiv d H_{a}^{+}=0
$$


Besides the space-time symmetries, considered in the previous subsection, equation (6.18) have internal rigid symmetries corresponding to the general linear transformations in the target space of fields:

$$
H_{a}^{+} \rightarrow G_{a}^{b} H_{b}^{+}, \quad\left(G_{a}^{b}\right) \in \mathrm{GL}(N, \mathbb{R})
$$

The case of two dimensions is rather special at least for three reasons. First, the subspaces of self-dual and anti-self-dual 1-forms admit a nice geometric visualization as a pair of transversal isotropic distributions defining the light cone at each point of $\mathbb{R}^{1,1}$. Second, the dynamical fields $H_{a}^{+}$are conserved current themselves for if we set $j_{a}=H_{a}^{+}$, then

$$
d j_{a}=T_{a} \approx 0
$$

Third, and most important, the Lagrange anchor (6.15) admits a non-abelian generalization 7 , which can be used to connect some of the rigid symmetries (6.19) with the conservation laws (6.20). To define this generalization we interpret the $N$-dimensional internal space of fields $H^{+}$as the linear space underlying a semi-simple Lie algebra $\mathcal{G}$ with a bases $\left\{t^{a}\right\}$ and the commutation relations

$$
\left[t^{a}, t^{b}\right]=f_{c}^{a b} t^{c}
$$

(Of course, such an interpretation imposes certain restrictions on the possible values of $N$. For example, it excludes $N=2$.) Then we can combine the fields $H_{a}^{+}$into a single $\mathcal{G}$-valued 1-form $H^{+}=H_{a}^{+} t^{a}$ subject to the equation of motion $d H^{+}=0$. Geometrically, one can think of $H^{+}$as a section of the trivial $S O(1,1) \times G$-vector bundle over $\mathbb{R}^{1,1}$, where $G$ is a Lie group with the Lie algebra $\mathcal{G}$.

Consider now the following non-abelian generalization of the Lagrange anchor (6.15):

$$
\langle V(W), P\rangle \doteq\left\langle W, V^{*}(P)\right\rangle=\left(W^{a}, d P_{a}+g\left[P, H^{+}\right]_{a}\right)
$$

Here $P=P_{a} t^{a}$ is a $\mathcal{G}$-valued anti-self-dual 1-form and $g$ is an arbitrary constant; all the Lie algebra indices are raised and lowered with the help of the Killing metric on $\mathcal{G}$. In case $g=0$, we have $N$ copies of the Lagrange anchors 6.15). For $g \neq 0$, the Lagrange structure remains integrable, but the corresponding Lie bracket (5.3) on the dual of the dynamics bundle becomes nontrivial:

$$
\left[e_{a}(x), e_{b}\left(x^{\prime}\right)\right]=-g f_{a b}^{c} e_{c}(x) \delta^{2}\left(x-x^{\prime}\right), \quad e_{a} \in \Lambda^{2}\left(\mathbb{R}^{1,1}\right) .
$$

\footnotetext{
${ }^{7}$ It should be stressed that this generalization has nothing to do with a non-abelian deformation of the original (abelian) equations of motion (6.18); the field equations remain the same.
} 
The conserved currents (6.20) correspond to the $\mathcal{G}$-valued characteristic $\Psi=-* \varepsilon_{a} t^{a}$ :

$$
\langle\Psi, T\rangle=-\left(* \varepsilon^{a}, d H_{a}^{+}\right)=\int_{X} d j, \quad j=\varepsilon^{a} H_{a}^{+} .
$$

By Proposition 5.1 this characteristic generates a proper symmetry transformation

$$
\delta_{\varepsilon} H^{+}=V(\Psi)=-g\left[\varepsilon, H^{+}\right], \quad \varepsilon=\varepsilon_{a} t^{a} .
$$

Thus, we see that the proper symmetries constitute a subgroup $\operatorname{Ad}(G) \subset \mathrm{GL}(N, \mathbb{R})$ in the group of all internal symmetries (6.19) of the equations of motion.

As to the relativistic symmetries of the system (6.18), these are proper and have the same form as in the abelian case (6.17).

\section{Conclusion}

Let us make some concluding remarks on the paper results and notice the open questions left for the future studies.

In the work [7], the concept of Lagrange anchor was introduced to formulate the pathintegral quantization for not necessarily Lagrangian field theories. In the present paper, we show that another important property of the Lagrangian dynamics - the relationship between rigid symmetries and conservation laws - extends to non-Lagrangian field equations whenever they are endowed with a Lagrange anchor. It was also shown in [7] that every Lagrange anchor gives rise to and can be related with a certain BRST complex on the ghost-extended configuration space of fields. In the Lagrangian gauge theory, this complex boils down to the well-known BRST complex associated with the Batalin-Vilkovisky master action. The unique existence of the local BV master action was proven long ago [33] under the assumptions that the original Lagrangian was local and the gauge symmetry was finitely reducible. Unlike the Lagrangian theory, the locality of both the Lagrange anchor and the non-Lagrangian equations does not necessarily result in the locality of the corresponding quantum BRST differential. Given a local Lagrange anchor for local field equations, the obstructions to the existence of a local BRST complex will be studied in our next paper. Remarkably, these obstructions, being crucial for the quantum theory, do not matter for the study of classical dynamics. At the classical level, the existence of a local Lagrange anchor is sufficient to link the conservation laws with rigid symmetries, as it is seen from the present paper. 
In the next work we also plan to develop the cohomological description of the rigid symmetries and conservation laws, making use of the BRST formalism for non-Lagrangian gauge theories [7]. This will extend the cohomological formulation of the Noether theorem, well studied in the Lagrangian setting [5], to not necessarily Lagrangian dynamics. In the entire BRST cohomology, we will identify the characteristic cohomology as well as the cohomology group capturing the rigid symmetries of non-Lagrangian equations of motion. Unlike the Lagrangian theory, these two groups of the BRST cohomology are not to be generally isomorphic to each other.

One can see that many important ingredients of the Lagrangian dynamics split into different categories in the non-Lagrangian setting. The local Lagrange anchor connects these categories, although not always making them identical. As a result, the Lagrange anchor endows the non-Lagrangian local field theory with some important features of the Lagrangian one. In particular, it offers a path-integral quantization of classical dynamics and establishes a (partial) connection between the rigid symmetries and the conservation laws.

\section{References}

[1] I.A. Batalin and G.A. Vilkovisky, Relativistic S-matrix of dynamical systems with boson and fermion constraints, Phys. Lett. B69 (1977) 309; I.A. Batalin and E.S. Fradkin, A generalized canonical formalism and quantization of reducible gauge theories, Phys. Lett. B122 (1983) 157.

[2] I.A. Batalin and G.A. Vilkovisky, Gauge algebra and quantization, Phys. Lett. B102 (1981) 27; Quantization of gauge theories with linearly dependent generators, Phys. Rev. D28 (1983) 2567; Existence theorem for gauge algebra, J. Math. Phys. 26 (1985) 172.

[3] M. Henneaux and C. Teitelboim, Quantization of gauge systems (Princeton University Press, Princeton, NJ, 1992).

[4] M. Henneaux, Consistent interactions between gauge fields: The cohomological approach, Contemp. Math. 219 (1998) 93.

[5] G. Barnich, F. Brandt, and M. Henneaux, Local BRST cohomology in gauge theories, Phys. Rept. 338 (2000) 439. 
[6] S.L. Lyakhovich and A.A. Sharapov, BRST theory without Hamiltonian and Lagrangian, JHEP 0503:011.

[7] P.O. Kazinski, S.L.Lyakhovich, and A.A.Sharapov, Lagrange structure and quantization, JHEP 0507:076.

[8] A.S. Cattaneo and G. Felder, Relative formality theorem and quantisation of coisotropic submanifolds, Adv. in Math., 208 (2007) 521.

[9] S.L. Lyakhovich and A.A. Sharapov, Schwinger-Dyson equation for non-Lagrangian field theory, JHEP 0602:007.

[10] S.L. Lyakhovich and A.A. Sharapov, Quantization of Donaldson-Uhlenbeck-Yau theory, Phys. Lett. B656 (2007) 265.

[11] S.L. Lyakhovich and A.A. Sharapov, Quantizing non-Lagrangian gauge theories: An augmentation method, JHEP 0701:047.

[12] S.L. Lyakhovich and A.A. Sharapov, Normal forms and gauge symmetries of local dynamics, J. Math. Phys. 50 (2009) 083510.

[13] I. Kolar, P. Michor, and J. Slovak, Natural operations in differential geometry (Springer-Verlag: Berlin Heidelberg, 1993).

[14] D.J. Saunders, The geometry of jet bundles (Cambridge University Press, Cambridge, 1989).

[15] P.J. Olver, Application of Lie groups to differential equations (Springer-Verlag, New York, 1986).

[16] I.S. Krasil'shchik, V.V. Lychagin, and A.M. Vinogradov, Geometry of jet spaces and nonlinear differential equations (Gordon and Breach, New York, 1986).

[17] B. DeWitt, Dynamical theory of groups and fields (Gordon and Breach, New York, 1965).

[18] R.L. Bryant and P.A. Griffits, Characteristic cohomology of differential systems I. General theory, J. Am. Math. Soc. 8 (1995) 507.

[19] A.M. Vinogradov, The $\mathcal{C}$-spectral sequence, Lagrangian formalism, and conservation laws. I. The linear theory, J. Math. Anal. Appl. 100 (1984) 1; The $\mathcal{C}$-spectral sequence, Lagrangian formalism, and conservation laws. II. The nonlinear theory, J. Math. Anal. Appl. 100 (1984) 41. 
[20] G. Barnich and M. Henneaux, Isomorphisms between the Batalin-Vilkovisky antibracket and the Poisson bracket, J. Math. Phys. 37 (1996) 5273.

[21] G. Barnich and M. Grigoriev, A Poincaré lemma for sigma models of AKSZ type, $\backslash$ protect \vrule width0pt \protect \href $\{$ http://arxiv.org/abs/0905.0547\}\{a

[22] K. Mackenzie, General theory of Lie groupoids and Lie algebroids (Cambridge University Press, Cambridge, 2005).

[23] A. Cannas da Silva and A. Weinstein, Geometric models for noncommutative algebras (AMS, Providence, RI, USA, 1999).

[24] H. Sussmann, Orbits of families of vector fields and integrability of distributions, Trans. Amer. Math. Soc. 180 (1973) 171.

[25] M. Henneaux, B. Knaepen, and C. Schomblond, Characteristic cohomology of pform gauge theories, Commun. Math. Phys. 186 (1997) 137.

[26] N. Marcus and J.H. Schwarz, Field theories that have no manifestly Lorentz invariant formulation, Phys. Lett. B115 (1982) 111.

[27] R. Floreanini and R. Jackiw, Self-dual fields as charge density solitons, Phys. Rev. Lett. 59 (1987) 1873.

[28] M. Henneaux and C. Teitelboim, Dynamics of chiral (self-dual) p-forms, Phys. Lett. B206 (1988) 650 .

[29] B. McClain, Y.S. Wu, and F.Yu, Covariant quantization of chiral bosons and OSp (1,1-2) symmetry, Nucl. Phys. B343 (1990) 689.

[30] P.P. Srivastava, On a gauge theory of self-dual field and its quantization, Phys. Lett. B234 (1990) 93.

[31] P. Pasti, D. Sorokin, and M. Tonin, On Lorentz invariant actions for chiral p-forms, Phys. Rev. D55 (1997) 6292.

[32] M. Alexandrov, M. Kontsevich, A. Schwarz and O. Zaboronsky, The geometry of the master equation and topological quantum field theory, Int. J. Mod. Phys. A12 (1997) 1405.

[33] M. Henneaux, Spacetime locality of the BRST formalism, Commun. Math. Phys. 140 (1991) 1. 